\title{
Folksonomias e pós-verdade: desafios para a organização do conhecimento
}

\author{
Folksonomies and post-truth: challenges for the \\ knowledge organization
}

\begin{abstract}
Juliana de Assis a,*
RESUMO: O estudo buscou propor uma redefinição do conceito de folksonomia a partir da identificação e análise das características, relações e impactos da pós-verdade enquanto fenômeno na organização do conhecimento. A metodologia utilizada tomou como base uma investigação de cunho exploratório e qualitativo que foi composta pela integração entre a pesquisa bibliográfica e a abordagem terminográfica. Apresenta como foco de análise um corpus constituído por 120 artigos publicados no período de 2005 a 2021 em âmbito internacional. Seus resultados possibilitaram caracterizar o fenômeno da pós-verdade e suas implicações no campo da organização do conhecimento; viabilizaram uma reflexão sobre as bases teórico-metodológicas que instituem as folksonomias como sistemas de organização do conhecimento, o que culminou com a compreensão da folksonomia como um modo algoritmicamente dirigido de manifestação e imposição de significados e discursos a partir das estruturas relacionais viabilizadas pelas redes sociotécnicas em contexto digital.
\end{abstract}

Palavras-chave: Folksonomia; Pós-verdade; Organização do Conhecimento; Ontologia; Desinformação.

ABSTRACT: This study sought to propose a redefinition for the concept of folksonomy from the identification and analysis of the characteristics, relationships and impacts of the post-truth as a phenomenon in the knowledge organization. The methodology used was based on an exploratory and qualitative investigation that was composed by the integration between the bibliographic research and the terminographic approach. It presents as focus of analysis a corpus made up of 120 articles published in the period from 2005 to 2021 in an international scope. Its results made it possible to characterize the phenomenon of post-truth and its implications in the field of knowledge organization; made possible a reflection on the theoretical and methodological bases that establish folksonomies as knowledge organization systems, which culminated in the understanding of folksonomy as an algorithmically directed mode of manifesting and imposing meanings and discourses based on the relational structures provided by the sociotechnical networks in digital context.

Keywords: Folksonomy; Post-truth; Knowledge Organization; Ontology; Disinformation.

\footnotetext{
a Departamento Biblioteconomia, Universidade Federal do Rio de Janeiro, Rio de Janeiro, RJ, Brasil.

* Correspondência para/Correspondence to: Juliana de Assis. Endereço: Avenida Pasteur - 250, Bairro Botafogo, 22290240 - Rio de Janeiro, RJ - Brasil. E-mail: juliana.assis@facc.ufrj.br.
}

Recebido em/Received: 15/04/2021; Aprovado em/Approved: 16/06/2021.

Artigo publicado em acesso aberto sob licença CC BY 4.0 Internacional @)(i) 


\section{INTRODUÇÃO}

A pós-verdade tem sido predominantemente descrita na literatura como uma expressão, utilizada pela primeira vez por Steve Tesich em 1992 na revista The Nation, que ganha popularidade e atenção de pesquisadores das mais diversas áreas a partir do ano de 2016 com os principais acontecimentos políticos mundiais que caracterizaram o período e com o fato de ter sido destacada pelo Dicionário Oxford naquele ano.

A partir da definição estabelecida pelo Dicionário Oxford, compreende-se que o conceito de pós-verdade denota um contexto histórico, social e cultural em que as subjetividades e a dimensão emocional das análises feitas pelos indivíduos se sobrepõem às evidências factuais e científicas. Tal sobreposição se dá tanto nas ações de elaboração, busca, seleção e compartilhamento da informação; quanto na apropriação das informações a fim de gerar novos conhecimentos. A estrutura de redes e a ação dos algoritmos acentuam isso ao possibilitarem a imersão dos indivíduos em redomas ou bolhas informacionais que atuam limitando o acesso a opiniões e visões de mundo dissonantes.

A organização do conhecimento (OC) é um campo de estudos que na concepção de Hjørland (2008) aborda as atividades e processos de representação da informação realizados por múltiplos agentes, tais como: especialistas, algoritmos e leigos. Constitui-se em uma das áreas centrais para a compreensão das características, mecanismos e efeitos da pós-verdade visto que se encontra posicionada na passagem entre a modelagem e a aplicação de formas de se ver o mundo. É detentora da capacidade e da responsabilidade de arbitrar signos e significados e assim instituir discursos e práticas. Desse modo, intermedia tanto a disposição quanto o acesso à informação por meio de modelos conceituais explicitados e formalizados nos sistemas de organização do conhecimento (SOC).

Os SOC são agentes que se encontram inseridos em processos comunicativos com acentuadas dimensões semióticas e discursivas (Feinberg, 2010). No contexto da pósverdade, as folksonomias são os tipos de SOC que mais evidenciam isso, uma vez que, viabilizam a manifestação e a expressão de preferências e interesses de indivíduos, bem como de grupos políticos e econômicos por meio de uma prática classificatória personalizada que se desenvolve a partir de ambientes digitais colaborativos, que conforme Farooq et al (2007) e Cattuto (2006), possuem como unidade fundamental de informação a tríade: pessoas, tags e recursos informacionais.

A palavra folksonomia se origina de um neologismo cunhado no ano de 2004 por Thomas Vander Wal como um tipo de classificação feita pelas pessoas no contexto digital por meio da atribuição de palavras-chave a um determinado conteúdo, detentor de uma URL (Vander Wal, 2004).

O presente estudo parte da percepção de que a folksonomia e a pós-verdade possuem nas redes sociais digitais uma estrutura sociotécnica comum, fator que contribui para que sejam respectivamente abordadas enquanto: um fenômeno que maximiza o uso 
da linguagem natural para a representação da informação na primeira e um fenômeno que envolve a negação sistemática de fatos objetivos na segunda. Ambos são intensificados pelo efeito das práticas colaborativas e discursivas gerado pelas redes sociais em contextos digitais.

As reflexões aqui apresentadas se fundamentam em um estudo de natureza exploratória e qualitativa realizado com base na pesquisa bibliográfica e na análise terminográfica de um corpus composto por 120 artigos oriundos de periódicos e conferências, em língua inglesa, publicados no período de 2005 a 2021. A base de dados científica Scopus foi a fonte utilizada para a identificação e seleção dos artigos. Foram focos das análises: título, resumo, palavras-chave e referencial teórico. Os critérios utilizados na escolha dos artigos foram: autoridade (o alto nível de citação de um autor), saturação (a recorrência de um determinado conceito ao longo dos trabalhos), relevância e valor semântico das palavras-chave. Os termos "folksonomy" e "posttruth" foram utilizados nas estratégias de buscas, de modo isolado e de forma combinada e restritiva a partir do uso do operador booleano "AND”. A delimitação metodológica pela língua inglesa se justifica pelas dimensões e implicações mundiais dos fenômenos observados. Foram selecionados um total de 80 artigos a partir do termo "folksonomy" e 40 a partir do termo "post-truth".

O estudo buscou cumprir os objetivos prévios de: 1) apontar características e impactos da pós-verdade no âmbito da OC; 2) sugerir contribuições oriundas desse campo para o enfrentamento da desinformação enquanto fenômeno informacional que se amplifica no contexto da pós-verdade; 3) propor uma redefinição para o conceito de folksonomia nesse cenário.

\section{FOLKSONOMIAS E PÓS-VERDADE: CONCEITOS, RELAÇÕES E IMPLICAÇÕES}

Nos últimos 20 anos mudanças significativas foram implementadas nas formas de criação, acesso, compartilhamento e uso dos objetos informacionais em contextos digitais. Isso alterou a relação dos sujeitos com a informação (aqui abordada em suas três acepções básicas: como documento, como significado e como um construto social) e com os seus espaços de mediação.

As folksonomias surgem no âmbito da Web 2.0, uma fase da web descrita por O'Reilly (2005) como um divisor de águas no modo de concepção dos padrões de software ancorado em um modelo de negócios baseado na radicalização do potencial da colaboração e da interação em rede e no máximo aproveitamento da inteligência coletiva.

A aparente autonomia conferida aos usuários das plataformas digitais na produção, organização e compartilhamento dos conteúdos e objetos informacionais é um fator que contribuiu para o enfraquecimento e descrédito dos veículos tradicionais de comunicação e informação e, por consequência, para o surgimento do fenômeno da pós-verdade, conforme destacam autores como McIntyre (2018) e Araújo (2020) ao refletirem sobre as suas causas. 
Segundo Mousselly-Sergieh et al (2014) a folksonomia é uma estrutura relacional composta por recursos informacionais, tags e usuários que possibilita a organização, navegação e recuperação de conteúdos na web. Nela observa-se a facilidade de acesso aos conteúdos, posto que são sistematizados de modo personalizável, por meio da linguagem natural.

Na literatura produzida nos últimos 16 anos de pesquisas sobre folksonomias é possível destacar a percepção comum de uma estrutura onde relações e fluxos geram esquemas interpretativos que refletem visões de mundo. Ao viabilizarem uma relação pessoal com os conteúdos e objetos informacionais as folksonomias entram no escopo dos estudos atuais sobre desinformação e pós-verdade.

De acordo com Freelon (2017) é possível observar uma relação intrínseca entre os ambientes digitais que na contemporaneidade promovem a crescente personalização da recuperação e do acesso à informação e a promoção da desinformação.

Compreende-se aqui o conceito de desinformação como um tipo de informação, caracterizada pela imprecisão e por ser alvo de criação e compartilhamento com a intencionalidade de causar engano, manipulação e prejuízos em relação a outros (Fallis, 2015; Wardle, Derakhshan, 2017). Nesse sentido, as notícias falsas (fake news) são abordadas como um termo mais específico, situado no campo semântico de um termo mais amplo que é a desinformação.

Tandoc Junior, Lim e Ling (2018) apud Estrada-Cuzcano, Alfaro-Mendives e SaavedraVásquez (2020, p. 99) apontam seis tipologias de fake news na literatura científica: 1) sátira: que constitui um conteúdo informativo repleto de humor ou exagero; 2) paródia: se diferencia da sátira por usar informações não objetivas para promover um conteúdo informativo que é caracterizado pelo humor a fim de levar a ridicularização; 3) fabricação: um conteúdo não baseado em fatos, porém publicado com feições de notícias de maneira que aparente ser legítimo; 4) manipulação: um conteúdo composto por notícias visuais mas com adulterações em seu significado; 5) publicidade: conteúdo informativo produzido com a finalidade vender ou promover produtos, empresas ou mesmo ideias; e 6) propaganda: um conteúdo informativo criado por entidades ou grupos políticos a fim de influenciar a percepção do público sobre um determinado assunto, pessoa ou instituição. $O$ quadro 1 sistematiza tais tipologias conforme o nível de sua factibilidade ou relação com fatos e eventos objetivos e ainda de acordo com o nível da intencionalidade em promover a enganação.

Destacam-se nas tipologias de fake news as diferentes formas de aproximação ou de distanciamento da realidade e o objetivo comum de enganar aqueles que as acessam impactando de modo prejudicial os comportamentos e os processos de tomada de decisão. 
Quadro 1. tipologias de definições de fake News.

\begin{tabular}{|lll|}
\hline \multirow{2}{*}{ Nível de factibilidade } & \multicolumn{2}{l|}{ Intenção imediata de enganar } \\
Alto & Alta & Baixa \\
& Publicidade & Sátira \\
& Propaganda & \\
Manipulação & Paródia \\
\hline
\end{tabular}

Traduzido e adaptado de Tandoc Junior, Lim e Ling (2018, p.12) apud Estrada-Cuzcano, AlfaroMendives e Saavedra-Vásquez (2020, p. 100)

Os ambientes digitais que adotam a folksonomia, enquanto propositores de estruturas relacionais e semióticas, contribuem para a geração de contextos ideais, tanto para a criação, descrição e compartilhamento de conteúdos e objetos informacionais fidedignos, quanto para o impulsionamento das várias modalidades de fake news e desinformação.

Nesse panorama, as tags expressam narrativas e discursos que, uma vez instituídos por perfis de pessoas e ainda por chatbots ${ }^{1}$, podem ser artificialmente modelados para manifestar, ou mesmo impor, visões de mundo.

Kalil e Santini (2020, p. 6) destacam que "[...] o uso de robôs nas redes sociais direciona a conversação online, rouba a atenção dos usuários, e funciona como uma nova forma de agendamento da imprensa e cria uma opinião pública artificial". Isso ocorre porque os robôs podem ser programados para realizarem um padrão de postagens que fazem com que determinadas tags adquiriram rápida popularidade na rede ou ainda percam popularidade e assim deixem de ser utilizadas pelos usuários.

A partir da observação das dinâmicas que perpassam esses ambientes, entende-se que há uma dimensão discursiva a ser estudada nos modos de criação e disseminação de todas as formas de desinformação. As folksonomias podem contribuir significativamente para a dispersão da mentira e do engano por meio dos discursos instituídos pelas tags.

Saquete et al (2020) apontam a disseminação de fake news como o principal motor da pós-verdade. Na visão desses autores, a pós-verdade é um fenômeno de distorção que visa manipular a opinião pública e o comportamento dos indivíduos.

A pós-verdade tem como base a exploração dos efeitos da mentira e do engano a partir da imposição de discursos por meio da comunicação mediada por computador (CMC) em sites de redes sociais. A mentira viralizada pelas plataformas digitais passa a atuar como um instrumento altamente eficiente de desinformação e gera um potencial significativo para a implementação de formas inovadoras de censura e controle (Magallón Rosa, 2019).

\footnotetext{
${ }^{1}$ Ferramenta de Inteligência Artificial (IA) criada com objetivo de simular um perfil de usuário humano nas interações realizadas via mídias sociais.
} 
Observa-se um contexto histórico, social e cultural em que o avanço das tecnologias de comunicação e informação proporciona múltiplas formas de organização e difusão da mentira e do engano conforme os interesses de grupos econômicos e políticos.

Barzilai e Chinn (2020) apontam cinco características da pós-verdade:

1) Aumento da predominância e influência da misinformation e da desinformação: As autoras utilizam as definições de Wardle e Derakhshan (2017) para diferenciar esses dois conceitos da seguinte maneira: misinformation é uma informação parcial ou totalmente incorreta que não intenta necessariamente enganar a outros e desinformação é uma informação imprecisa disseminada com a intenção de manipular ou prejudicar a outros.

2) Crescente rejeição ao conhecimento pré-estabelecido a partir de fatos e análises científicas: nesse cenário o conhecimento científico perde espaço para teorias da conspiração e fontes de informação não especializadas.

3) Estabelecimento de conviç̧ões e experiências pessoais acima dos fatos e evidências: a dimensão pessoal passa a ser o principal parâmetro para a leitura da realidade.

4) Declínio da confiança nas fontes institucionais da informação: entidades científicas e jornalísticas perdem espaço para redes e plataformas personalizáveis de informação.

5) Aumento da fragmentação e polarização do consumo da informação: cenário caracterizado pelas bolhas informacionais geradas a partir da segmentação de conteúdos promovida pelas estruturas algorítmicas.

Percebe-se que as cinco características da pós-verdade se interrelacionam de modo a formarem um contexto denso, constituído por inúmeras variáveis e fatores condicionantes.

Barzilai e Chinn (2020) citam ainda fatores sociotécnicos, políticos, econômicos e científicos articulados na catalização do fenômeno da pós-verdade e salientam que outros fatores de cunho psicológico e educacional interagem com esses na sua configuração como uma condição contemporânea.

A dimensão informacional da pós-verdade demanda ser analisada e descrita pela ciência da informação $(\mathrm{Cl})$ em diálogo com todos os fatores que a constituem. Sob essa perspectiva, o núcleo informacional do fenômeno enseja estudos que vão desde os fundamentos epistemológicos da $\mathrm{Cl}$ às novas ecologias informacionais, com especial atenção às formas de organização, recuperação e uso dos conteúdos e objetos informacionais.

No campo da organização do conhecimento as contribuições para o entendimento da pós-verdade e o enfrentamento de suas consequências tendem a acentuar a tensão entre ética e tecnologia. Tais tensões precisam evoluir para uma compreensão teórica e prática sobre a indissociabilidade entre esses dois pilares. 
Saquete et al (2020) observam que a linguagem natural é a principal forma de formalização e disseminação de conteúdos e objetos informacionais ao longo das redes sociais digitais e destacam o potencial do uso da inteligência artificial nos esforços para identificação automática das fake news. Os autores propõem uma metodologia baseada nas seguintes subtarefas: 1) detecção de engano; 2) detecção de posição; 3) controvérsia e polarização; 4) verificação automatizada de fatos; 5) detecção de clickbait²; e 6) pontuações de credibilidade. As seis subtarefas pressupõem o uso de massivo de técnicas de processamento da linguagem natural.

Observa-se ainda na literatura o nascedouro de pesquisas como Groza (2020) que intentam promover a aplicação das ontologias para a geração de inferências que possibilitem a detecção de fake news.

Os estudos de Saquete et al (2020) e Groza (2020) exemplificam como a tecnologia pode ser aplicada para combater os efeitos da pós-verdade, mas, sem considerarmos a sua dimensão ética podem se tornar ineficazes pois a condição contemporânea evidencia como o avanço tecnológico promove e amplifica a desinformação.

Em consonância com os estudos citados, Paula, Silva e Blanco (2018) apontam a urgência de criação de estruturas dedicadas à auditoria sistemática dos conteúdos disseminados pelas redes sociais em contexto digital. Mas, além disso, as legislações dos países precisam abarcar essa realidade garantindo que empresas de tecnologia (especialmente as big techs ${ }^{3}$ ) cumpram preceitos éticos mínimos ao propor e gerenciar as ações tantos dos seus usuários, quanto dos seus algoritmos. Pessoas físicas e jurídicas devem estar sujeitas às leis que considerem a dimensão ética da criação, do uso e da disseminação dos conteúdos e objetos informacionais na web.

Observa-se na literatura três esferas básicas de atuação a serem consideradas nesse âmbito: 1) educação; 2) legislação e 3) auditoria dos conteúdos.

$\mathrm{Na}$ esfera educacional os estudos sobre competência em informação devem somar esforços com as pesquisas específicas em tagging literacy a fim de cultivar a "[...] sensibilização e formação humana para o desenvolvimento de atividades de classificação da informação e do conhecimento em ambientes colaborativos digitais" (Mejias, 2005 apud Moura, 2009, p. 34).

No cenário da pós-verdade, a tagging literacy é um tipo de competência que pode contribuir para que os sujeitos informacionais tenham a capacidade de identificar discursos artificialmente promovidos como hegemônicos em ambientes que adotam a folksonomia. A partir desse tipo de literacia evidencia-se como a livre atribuição de tags, por indivíduos e ainda por inteligências artificiais, pode atuar tanto na

\footnotetext{
2 Em sua tradução literal: "caça-clique”, trata-se de um conteúdo com título chamativo ou apelativo que promove o acesso a propagandas e notícias imprecisas ou falsas e geram a sua propagação pela rede mediante as dinâmicas de compartilhamento.

3 Termo utilizado para definir as grandes empresas de tecnologia que atuam com base na monopolização dos dados dos usuários da web. São exemplos de big techs: Amazon, Facebook e Google.
} 
disseminação de conteúdos e objetos informacionais dotados de qualidade e credibilidade quanto na promoção da desinformação.

$\mathrm{Na}$ esfera das legislações, observa-se que iniciativas efetivas para a regulação da atuação das big techs são escassas e recentes. Revelam as dificuldades que países e blocos regionais precisam superar nesse âmbito.

No Brasil, por exemplo, em julho de 2020, foi apresentado o Projeto de Lei (PL) 2630/2020 que pretende instituir a "Lei Brasileira de Liberdade, Responsabilidade e Transparência na Internet", conhecida como "Lei das Fake News". Após aprovação no Senado Federal, o PL segue até o momento (segundo trimestre de 2021) em tramitação na Câmara dos Deputados sendo foco de significativos esvaziamentos em seu conteúdo de modo a comprometer a sua efetividade no combate aos aparatos de desinformação.

O presente trabalho toma como modelo a legislação proposta pela União Europeia devido ao seu escopo, abrangência e sintonia em relação aos problemas e desafios aqui abordados.

Em dezembro de 2020 a União Europeia apresentou um proposta de regulamentação composta pela DSA (Digital Services Act) e pela DMA (Digital Markets Act) que possuem como objetivos, respectivamente: "criar um espaço digital mais seguro no qual os direitos fundamentais de todos os usuários de serviços digitais sejam protegidos" e "estabelecer condições de concorrência equitativas para fomentar a inovação, o crescimento e a competitividade, tanto no Mercado Único Europeu como a nível mundial" (European Commission, on-line, tradução nossa).

A DSA especificamente contempla uma regulamentação direcionada ao combate à desinformação promovida tanto por empresas responsáveis pelas plataformas digitais, quanto por seus usuários. Abarca ainda a responsabilização dessas empresas pela ação dos seus algoritmos e sistemas de recomendação trazendo diretrizes para a explicitação dos seus mecanismos de atuação. É possível destacar nessa legislação um considerável avanço em relação à consciência que os legisladores possuem sobre os impactos da ação de tais mecanismos.

Uma parte essencial da atividade de uma plataforma on-line de grande dimensão se relaciona à forma como a informação é priorizada e apresentada na sua interface para facilitar e otimizar o acesso dos usuários do serviço. Isso feito, por exemplo, através da sugestão, classificação e priorização algorítmica da informação, de uma distinção mediante texto ou de outras representações visuais, ou da conservação da informação fornecida pelos usuários. Estes sistemas de recomendação podem ter um impacto significativo na capacidade dos usuários de recuperar e interagir com a informação on-line. Desempenham igualmente um papel importante na amplificação de determinadas mensagens, na propagação viral da informação e no estímulo do comportamento on-line (European Commission, 2020, p. 37, tradução nossa). 
Os legisladores europeus apresentaram aos países membros da União Europeia uma Lei de Serviços Digitais que pode ser utilizada como modelo a ser adaptado inclusive por países como o Brasil ao reconhecerem e apontarem como o modo de atuação das big techs influencia ou determina não somente o que seus usuários acessam na rede mas sobretudo o comportamento a ser desencadeado a partir do acesso e da apropriação das informações, caracterizando o que Morozov e Marcondes (2018) denominaram regulação algorítmica.

Na esfera da auditoria de conteúdos, observa-se a necessidade de uma atuação multidisciplinar e com a participação de diferentes atores da sociedade, dentre os quais se destacam as próprias big techs com o cumprimento de regulamentações que resultem em reavaliações e mudanças em seus modelos de negócios.

A OC possui um relevante papel a ser desempenhado na proposição de estruturas informacionais que viabilizem uma web de conteúdos auditáveis potencializando práticas como a fact-checking 4 .

Observa-se que o avanço das pesquisas sobre folksonomia, especialmente em EUA e China, países que mais desenvolvem estudos científicos sobre o tema segundo levantamento realizado em 2021 na base de dados Scopus, indicam um inescapável alinhamento entre folksonomias e ontologias a fim de promover sistemas de recomendação que possibilitem melhorias na recuperação da informação em múltiplos campos e contextos de aplicação (Nauman; Hussain, 2007; Vig, 2010; Qassimi, Abdelwahed, 2019).

A ontologia é um tipo de $\mathrm{SOC}$ que preconiza a explicitação e formalização dos conceitos e suas relações no nível dos sistemas e aplicações computacionais visto que "requer uma possibilidade de interpretação algorítmica dos seus significados" (Feitosa, 2006, p.73). Uma vez aliada ao potencial de atualização terminológica e de contextualização que as folksonomias viabilizam, as ontologias podem enriquecer os ambientes digitais colaborativos com uma estruturação do conhecimento passível de inferências.

Nesse sentido, o conceito de ontologia folksonomizada revela um SOC que hibridiza a linguagem natural e a linguagem controlada agregando os aspectos positivos de ambas a fim de promover avanços na estrutura lógica que embasa a representação e a recuperação dos conteúdos e objetos informacionais, conforme evidenciam os estudos de Alves e Santanchè (2011); Wang et al. (2015); Yadav et al. (2016) e Salgado (2019).

Uma aplicação possível das ontologias folksonomizadas no contexto da pós-verdade teria como foco principal a identificação de fake news o que uma vez ancorado na dimensão ética e social da OC possibilitaria a criação e a disseminação de técnicas de recomendação de tags e conteúdos baseadas em inteligência artificial e

\footnotetext{
${ }^{4}$ Termo utilizado para denotar a prática de checagem de fatos, dados e informações conforme
} as evidências e fontes fidedignas. 
compromissadas com a veracidade dos fatos e com as evidências científicas, atuando de modo integrado com sites e serviços de fact-checking.

\section{FOLKSONOMIAS: COMPONENTES DA PÓS-VERDADE?}

Folksonomia e pós-verdade são conceitos relacionados na contemporaneidade. $O$ estudo destaca a incipiência das pesquisas que relacionam diretamente os dois conceitos e a urgência de novas investigações, principalmente sob a ótica da $\mathrm{Cl}$ e especificamente da OC.

As buscas restritivas realizadas com o operador booleano AND e os termos "folksonomy" e "post-truth" não apresentaram resultados significativos, retornando apenas um artigo publicado em 2020 na Rússia. Nele Ryabchenko e Malysheva (2020) abordam as características da comunicação política moderna nos ambientes digitais. $O$ que revela como o caráter discursivo e passível de modulação algorítmica dos ambientes folksonômicos tornou-se uma seara propícia para a promoção de discursos de cunho político e ideológico.

No tocante às buscas com o termo "folksonomy", observou-se na literatura analisada a configuração de duas grandes frentes de investigação não excludentes: uma dedicada aos aspectos semânticos dos ambientes folksonômicos com destaque para pesquisas que visam aprimorar a recomendação de tags e conteúdos informacionais e outra dedicada a inserir nesses ambientes colaborativos uma estruturação lógica e formal, que faça com que inferências possam ser realizadas.

A figura 1 sintetiza os principais conceitos identificados a partir da análise do corpus da pesquisa. Foi utilizada a nuvem de tags como um recurso gráfico que possibilita destacar os conceitos mais recorrentes e ainda os de maior valor semântico.

Figura 1. Nuvem de conceitos gerada a partir do termo "folksonomy".

\section{Collaborative filtering Recommender systems Collaborative tagging Recommendation Technologies User Modeling Link Analysis Social Knowledge} Complex systems Emergent semantics Tagging Information Analysis Data reduction Computer supported cooperative work Knowledge representation Folksonomy system Graph theory Ontologies Artificial intelligence Information sources Semantic infrastructure Semanticweb Bookmarks Metadata Mathematical models Pattern recognition Clustering Personalization Reducing noise Semantic similarity Word embedding Algorithms community identification algorithms knowledge extraction Automatic tagging Semantics in folksonomy Folksonomy enrichment Search precision Cognitive information Tag relatedness Content Analysis Image indexing Behavioral research Classification Probability distribution Tagging status Natural language processing systems Thesauri Statistical language modeling Tag recommendation User interfaces Hybrid recommenders Recommendation techniques Annotation systems folkrank Fonte: dados da pesquisa. 
Considera-se que as tags não expressam apenas conteúdos, mas discursos que contribuem para a validação e propagação da informação (e da desinformação) conforme os perfis de interesse.

Na era da pós-verdade, a folksonomia se configura como um modo algoritmicamente dirigido de manifestação e imposição de discursos e significados que viabiliza a propagação segmentada de conteúdos e objetos informacionais. Esta proposta de definição conceitual não exclui as definições existentes na literatura, apenas amplia o entendimento que se tem das folksonomias enquanto tipos de SOC.

O nível de personalização da relação com a informação que as folksonomias viabilizam demanda uma abordagem vigilante da $O C$, sobretudo no que concerne à sua capacidade de gerar e ampliar bolhas informacionais dado o alto potencial de manifestação e imposição de discursos enviesados pela ação, não somente dos sujeitos, mas também dos dispositivos algorítmicos que arbitram as plataformas digitais.

Nelas, sujeitos informacionais são detentores de certa autonomia para indexar seus conteúdos e objetos informacionais de acordo com seus pontos de vista e necessidades. Contudo, é pertinente considerarmos que essa autonomia não é total e nem isenta da ação de inteligências artificiais e da curadoria algorítmica que caracteriza esses ambientes digitais.

As buscas com o termo "post-truth" recuperaram trabalhos científicos dedicados à descrição do fenômeno da pós-verdade e de seus conceitos relacionados. A figura 2 sistematiza os principais conceitos abordados nesses estudos.

Figura 2. Nuvem de conceitos gerada a partir do termo "post-truth".

COVID-19 Equilibrium model Policy sciences Brexit Democracy Digital media Digital political marketing Far right Digital transformation Conspiracy Theories Critical theory Critique emancipation Ideology Truth Post-truth politics Algorithmic culture Attention Cultural studies Deep fakes Digital after life Industry everyday Theory postdigital studies Data quality assurance Open data Risk identification Risk mitigation Echo chambers aging Fake news Misinformation Epistemic bubbles Conceptual truth Emergent meanings Semantic change Fake science Loss of meaning of scientific truth Post-truth era Scientific fraud Value-based responsibility Communication Journalistic language Persuasion Pragmatics Anti-politics Discourse analysis Rhetoric Belief information Post-modernism Post-truth claims Social media Automatic fact-checking Clickbait detection Credibility Deception detection Human language Technologies Natural Language Processing Stance detection Inference Bias Illusory truth Social networks Emotional reporting Information selection Social cue Narrative paradigma Trump Agnotology Semiotics Discursive regime Media literacy Informational literacy

Fonte: dados da pesquisa. 
Destaca-se nesse panorama o papel ativo de mecanismos que instituem uma pervasiva curadoria algorítmica que atua na filtragem e direcionamento de conteúdos e contribui para que os sujeitos informacionais lidem com a exponencial massa de dados e informações disponíveis na web ao mesmo tempo em que fornece as bases informacionais da pós-verdade.

Percebeu-se que as folksonomias não figuram entre os componentes da pós-verdade, não se trata de uma relação todo-parte, mas de uma possível relação de causalidade visto que, enquanto SOC baseados em linguagem natural, encontram-se inseridas na densa cultura informacional formada pela interação dos fatores e condicionantes que juntos instituem a Pós-verdade.

As tags e a modulação algorítmica a que estão suscetíveis instauram uma retórica digital maleável conforme os interesses de grupos políticos e econômicos que retroalimenta um ciclo de representação, busca e recuperação personalizável da informação. Considera-se relevante salientar aqui que a desinformação também é informação (FALLIS, 2015). Sendo assim, as práticas de classificação distribuída realizadas a partir da atribuição de tags ao longo das redes sociais digitais contribuem para a ocorrência do fenômeno informacional em todas as suas dimensões e impactos.

\section{CONCLUSÕES}

A personalização da relação com a informação que as folksonomias propiciam instigam uma abordagem crítica e ética da OC em relação: a) aos múltiplos atores que arbitram e modelam essa linguagem; b) ao seu potencial de desencadear e amplificar as bolhas informacionais; e c) à sua capacidade de promoção das várias formas de desinformação.

Esforços interdisciplinares empreendidos nesse campo podem auxiliar na descrição e compreensão dos fenômenos informacionais contemporâneos, além de contribuírem para o enfrentamento da desinformação, mitigando seus impactos a partir de modelos conceituais formais que viabilizem uma web em que as inferências e as recomendações promovidas pelos mecanismos que instauram a curadoria algorítmica vigente cooperem para o reestabelecimento da verdade, a ser alcançada sob uma perspectiva objetiva, pautada pela valorização dos fatos e evidências científicas.

\section{REFERÊNCIAS}

ALVES, Hugo.; SANTANCHÈ, André, 2011. Folksonomized Ontologies-from social to formal. In: XVII Brazilian symposium on multimedia and the web. 2011, p. 58-65.

ARAÚJO, C. A. V., 2020. O fenômeno da pós-verdade e suas implicações para a agenda de pesquisa na ciência da informação. Encontros Bibli: Revista Eletrônica de Biblioteconomia e Ciência da Informação [em linha]. 2020, v. 25, p. 1-17, 2020. [Acesso em 05 março 2021]. Disponível em: https://doi.org/10.5007/1518-2924.2020.e72673. 
BARZILAI, S.; CHINN, C., 2020. A review of educational responses to the "post-truth" condition: four lenses on "post-truth" problems. Educational Psychologist [em linha]. 2020. v. 55. n. 3, p. 107-119. [Acesso em 20 março 2021]. Disponível em: https://www.tandfonline.com/doi/full/10.1080/00461520.2020.1786388.

CATUTTO, Ciro, 2006. Semiotic dynamics in online social communities. The European Physical Journal C. 2006, p. 33-37.

EUROPEAN COMMISSION, 2020. Proposal for a regulation of the european parliament and of the council on a Single Market For Digital Services (Digital Services Act) and amending Directive 2000/31. [em linha]. 2020. [Acesso em 30 março 2021]. Disponível em: https://ec.europa.eu/digital-single-market/en/laws.

ESTRADA-CUZCANO; Alonso.; ALFARO-MENDIVES, Karen.; SAAVEDRA-VÁSQUEZ, Valeria, 2020. Disinformation y misinformation, posverdad y fake News Precisiones conceptuales, diferencias, similitudes y yuxtaposiciones. Información, cultura y sociedad [em linha]. 2020. [Acesso em 20 março 2021]. Disponível em: http://revistascientificas.filo.uba.ar/index.php/ICS/article/view/7427/7057.

FALLIS, Don, 2015. What's disinformation?. Library Trends [em linha]. 2015. v. 63, n. 3, p. 401-426. [Acesso em 8 março 2021]. Disponível em: https://www.ideals.illinois.edu/bitstream/handle/2142/89818/63.3.fallis.pdf.

FAROOQ, Umer, 2007. Evaluating tagging behavior in social bookmarking systems: metrics and design heuristics. International ACM SIGGROUP Conference on Supporting Group Work. 2007.

FEINBERG, Melanie, 2010. "Two kinds of evidence: how information systems form rhetorical arguments", Journal of Documentation [em linha]. 2010. v. 66 n. 4, p. 491-512. [Acesso em 8 março 2021]. Disponível em: https://doi.org/10.1108/00220411011052920.

FEITOSA, Ailton, 2006. Organização da informação na web: das tags à web semântica. Brasília, DF: Thesaurus. $131 \mathrm{p}$.

FREELON, Deen, 2017. Personalized information environments and their potential consequences for disinformation. In: Understanding and addressing the disinformation ecosystem, 2017. p. 5-12.

GROZA, Adrian, 2020. Detecting fake news for the new coronavírus by reasoning on the Covid-19 ontology [em linha]. 2020. [Acesso em 20 março 2021]. Disponível em: https://arxiv.org/pdf/2004.12330.pdf.

HJØRLAND, Birger, 2008. What is Knowledge Organization (KO)? Knowledge Organization [em linha]. 2008. v. 35, n. 3/2, p. 86-101. [Acesso em 10 março 2021]. Disponível em: https://www.ergon-verlag.de/isko_ko/downloads/k035200823.pdf.

KALIL, Isabela.; SANTINI, Rose Marie, 2020. Coronavírus, Pandemia, Infodemia e Política. Relatório de pesquisa. São Paulo / Rio de Janeiro: FESPSP / UFRJ. 2020.

MAGALLÓN ROSA, Raúl, 2019. Unfaking News: como combater a desinformação. Porto: Media XXI. 2019.

McINTYRE, L., 2018. Posverdad. Madrid: Cátedra, 2018. 
MEJIAS, Ulises, 2005. Tag literacy. [em linha] 2005. [Acesso em 20 março 2021]. Disponível em: https://blog.ulisesmejias.com/2005/04/26/tag-literacy/.

MOROZOV, Evgeny; MARCONDES, Claudio, 2018. Big tech: a ascensão dos dados e a morte da política. São Paulo: Ubu, 2018.192 p.

MOURA, Maria Aparecida, 2009. Folksonomias, redes sociais e a formação para o tagging literacy: desafios para a organização da informação em ambientes colaborativos virtuais. Informação \& Informação [em linha]. 2009. v. 14, n. 1esp, p. 2545. [Acesso em 20 março 2021]. Disponível em: http://www.uel.br/revistas/uel/index.php/informacao/article/view/2196.

MOUSSELLY-SERGIEH, H.; EGYED-ZSIGMOND, E.; GIANINI, G.; DÖLLER, M.; PINON, J.M.; KOSCH, H., 2014. Tag relatedness in image Folksonomies. Document Numerique [em linha]. 2014. v.17, n. 2, p. 33-54. Disponível em: https://www.cairn.info/revuedocument-numerique-2014-2-page-33.htm.

NAUMAN, Mohammad.; HUSSAIN, Fida., 2007. Common sense and folksonomy: Engineering an intelligent search system. International Conference on Information and Emerging Technologies [em linha]. 2007. p. 142-147. [Acesso em 30 março 2021]. Disponível em: https://ieeexplore.iee.org/document/4381326.

O'REILLY, Tim, 2005. What Is Web 2.0? Design Patterns and Business Models for the Next Generation of Software. [em linha]. 2005. [Acesso em 8 março 2021]. Disponível em: http://www.oreilly.com/pub/a/web2/archive/what-is-web-20.html.

PAULA, Lorena Tavares de.; SILVA, Thiago dos Reis Soares da.; BLANCO, Yuri Augusto, 2018. Pós-verdade e Fontes de Informação: um estudo sobre fake news. Revista Conhecimento em Ação [em linha]. v. 3, n. 1, 2018. p.93-110. [Acesso em 20 março 2021]. Disponível em: https://revistas.ufrj.br/index.php/rca/article/view/16764.

QASSIMI, S.; ABDELWAHED, E.H. The role of collaborative tagging and ontologies in emerging semantic of web resources. Computing. v. 101, n. 10, 2019, p. 1489-1511.

RYABCHENKO, Natalia Anatolievna.; MALYSHEVA, Olga Petrovna., 2020. Characteristic features of modern political communication in the online-space. Voprosy Kognitivnoy Lingvistiki, v. 2020, n. 2, 2020, p. 101-113.

SALGADO, Isa Lice da Mota Rodrigues, 2019. A model of folksonomized ontologies for auditing financial institutions in Brazil. CEUR Workshop. 2019.

SAQUETE, E.; TOMÁS, D.; MOREDA, P.; MARTÍNEZ-BARCO, P.; Palomar, M., 2020. Fighting post-truth using natural language processing: a review and open challenges. Expert Systems with Applications [em linha]. 2020. [Acesso em 20 março 2021]. Disponível em: https://www.sciencedirect.com/journal/expert-systems-withapplications.

TANDOC JR, Edson C., LIM, Zheng Wei, LING, Richard, 2018. Defining “Fake News": a typology of scholarly definitions. Digital Journalism [em linha]. 2018, v. 6. n. 2, p. 137153. [Acesso em 20 março 2021]. Disponível em: https://www.tandfonline.com/doi/full/10.1080/21670811.2017.1360143. 
VANDER WAL, Thomas, 2007. Folksonomy coinage and definition. [em linha]. 2007. [Acesso em 8 março 2021]. Disponível em: https://vanderwal.net/folksonomy.html.

VIG, Jesse, 2010. Intelligent tagging interfaces: beyond folksonomy. XXIII Annual ACM symposium on user interface software and technology. [em linha]. 2010. [Acesso em 30 março 2021]. Disponível em: https://dl.acm.org/doi/abs/10.1145/1866218.1866226.

WANG, S.; ZHUANG, Y.; HU, Z.; FEI, X., 2015. An ontology evolution method based on folksonomy. Journal of Applied Research and Technology. [em linha]. v. 13, n. 2, 2015, p. 177-187. [Acesso em 15 março 2021]. Disponível em: https://doi.org/10.1016/j.jart.2015.06.015.

WARDLE, Claire; DERAKHSHAN, Hossein, 2017. Information disorder: toward an interdisciplinary framework for researchand policy making. [em linha]. 2017. [Acesso em 30 março 2021]. Disponível em: https://rm.coe.int/information-disorder-toward-aninterdisciplinary-framework-for-researc/168076277c.

YADAV, Usha.; KAUR, Jaipreet.; DUHAN, Neelam., 2016. Semantically related tag recommendation using folksonomized ontology. III International Conference on Computing for Sustainable Global Development. 2016. 